\title{
Falsa creencia y desarrollo semántico del lenguaje en niños de 2 a 4 años*
}

\section{False Belief and Semantic Language Development in Children Aged 2 to 4 Years}

Recibido: febrero 27 de 2010 | Revisado: abril 10 de 2010 | Aceptado: abril 15 de 2010

\author{
Milton EduARdo BermúdeZ-Jaimes ** \\ Pontificia Universidad Javeriana, Bogotá, Colombia \\ Luz Viviana SASTRE-GÓMEZ \\ Universidad de Buenos Aires, Argentina
}

Para citar este artículo. Bermúdez-Jaimes, M.B. \& Sastre-Gómez, L.V. (2010). Falsa creencia y desarrollo semántico del lenguaje en niños de 2 a 4 años. Universitas Psychologica, 9 (3), 849-861.

* Artículo de investigación.

** Laboratorio de Psicología, Cr. 5 \# 39-00, oficina 110. Correo electrónico: milton.bermudez@javeriana.edu.co

\section{RESUMEN}

Se presentan los resultados de un estudio realizado con una muestra de 116 niños y niñas, con edades entre los 2 y los 4 años, de las ciudades de Bogotá y Chía, en donde se intenta explorar y caracterizar las relaciones existentes entre el desarrollo de la comprensión de las teorías infantiles de la mente y el desarrollo semántico del lenguaje. Para ello, se utilizaron tres versiones de la tarea de la falsa creencia digitalizadas mediante la aplicación Flash y la adaptación al castellano de la batería de evaluación de desarrollo temprano del lenguaje (Hresko et al., 1981) para evaluar las habilidades semánticas del lenguaje. Los hallazgos señalan diferencias significativas entre las edades, en términos de su nivel de desempeño para cada una de las tareas, así como las asociaciones positivas entre comprensión social y desarrollo semántico del lenguaje, para dos de las tres tareas propuestas. Los hallazgos se interpretan en el marco de la propuesta interaccionista desarrollada por Wellman (1994). Palabras clave autores

Cognición social, desarrollo semántico, falsa creencia, lenguaje, teorías infantiles de la mente.

Palabras clave descriptor

Niños (2-4 años), aspectos psicológicos, adquisición del lenguaje, aptitud verbal.

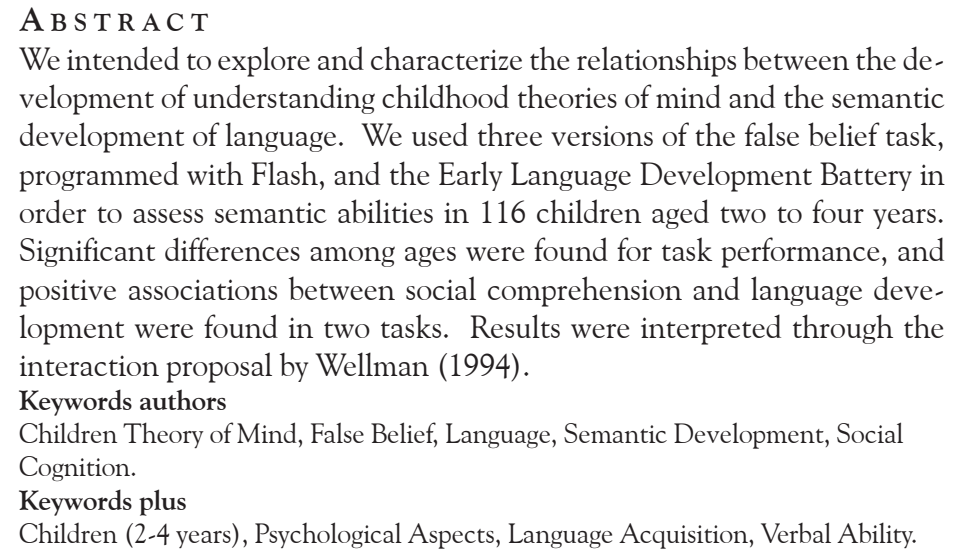

We intended to explore and characterize the relationships between the development of understanding childhood theories of mind and the semantic development of language. We used three versions of the false belief task, programmed with Flash, and the Early Language Development Battery in order to assess semantic abilities in 116 children aged two to four years. Significant differences among ages were found for task performance, and positive associations between social comprehension and language development were found in two tasks. Results were interpreted through the interaction proposal by Wellman (1994).

Keywords authors

Children Theory of Mind, False Belief, Language, Semantic Development, Social Cognition.

Keywords plus

Children (2-4 years), Psychological Aspects, Language Acquisition, Verbal Ability. 
La complejidad de la comunicación humana interpersonal exige del niño el despliegue de una serie de competencias que permiten atribuir a los otros deseos y creencias, mediante delicados procesos de adaptación a los procesos mentales de éstos (Antonietti, Liverta-Sempio \& Marchetti, 2005; Riviére \& Núñez, 1996; Wolfgang, Schumann-Hengsteler \& Sodian, 2004), pero la comprensión de la Teoría de la Mente no se da como un aparición absoluta y repentina, por el contrario, es una capacidad que se va construyendo con diferentes niveles de complejidad, siendo los cuatro años uno de estos momentos cuando el niño comprende el carácter representacional de los estados mentales.

Uno de los precursores importantes en el desarrollo de la Teoría de la Mente es el desarrollo de los patrones de referencia social y de la comprensión de las emociones. La referencia social está directamente relacionada con el desarrollo de los prerrequisitos de comunicación. Hacia los cinco meses de edad, el niño distingue expresiones emocionales, y antes de los nueve meses manipulará objetos e interaccionará con los demás, sonriendo y balbuceando. Hacia los dos años los niños se dan cuenta que son las creencias sobre una situación las que determinan una emoción y no la situación misma (Martí, 1997). Ligado a estas conquistas está el desarrollo de la comunicación. Uno de estos primeros momentos es la comunicación intencional que suele aparecer al final del primer año de vida (Astington, 1998; Astington \& Baird, 2005).

En la intención comunicativa temprana de los bebés, la señalización cumple en primera instancia una función imperativa; el bebé muestra al adulto algo con el deseo de pedirlo u obtenerlo, a la vez que manipula su conducta. Esta función recibe el nombre de "proto-imperativa" (Martí, 1997). Progresivamente, el acto de señalar trasciende a otra función esencial que recibe el nombre de "proto-declarativa", en la que el niño señala un objeto con el propósito de obtener la atención del adulto. Según el autor, estas distinciones tienen gran importancia, ya que, en el caso de la función declarativa, sugiere que el bebé sabe que la otra persona posee estados mentales, que los diferen- cia de los suyos y que puede incidir en los estados mentales ajenos para modificarlos en su favor.

A los dos años, el desarrollo del lenguaje y la aparición del juego simbólico permiten al niño la comprensión de pronombres personales como "tú", "yo" y los posesivos como "tuyo", "mío", en el contexto comunicativo, marcando con claridad el surgimiento de la autoconciencia. La progresiva adquisición del lenguaje oral contribuye al desarrollo de la Teoría de la Mente, y la utilización del lenguaje, como vehículo de comunicación, facilita el desarrollo de los aspectos pragmáticos (Astington, 1998; Astington \& Baird, 2005).

La comprensión del conocimiento por parte de los niños se ha estudiado (de forma empírica) de muchas maneras. Piaget mostró gran interés por explicar las características y la coherencia del pensamiento infantil, en diferentes estadios del desarrollo. Uno de los términos acuñados por Piaget (1929/) fue el de "realismo infantil"; con éste hace referencia al carácter realista del pensamiento infantil, describiendo la incapacidad cognitiva por parte del niño, para diferenciar los fenómenos mentales internos de los fenómenos físicos externos.

Otros estudios revelaron la manera en que los niños de dos años emplean los términos mentales como: saber, pensar, recordar, fingir, significar, olvidar, recordar y adivinar. Wellman y Estes (1986) encuentran que el uso cotidiano de los verbos mentalistas por parte de los niños, sugiere que se emplean en primera instancia para indicar referencia mental "probable", como por ejemplo: "Pensaba que no habían calcetines [en el cajón], pero cuando miré, los vi. No sabía que tú los tenias”. En estos casos, se puede apreciar que el niño contrasta lo real con lo mental, aclarando, para su interlocutor, interpretaciones objetivas erróneas, lo que indica que los niños distinguen lo mental de lo físico, en el uso de los términos mentales.

En sus primeras investigaciones Wellman y Estes (1986) indagaron si los niños de 3 a 5 años juzgaban las entidades físicas y mentales como diferentes. Para esto, evaluaron la comprensión de los niños acerca de las distinciones ontológicas entre 
entidades mentales y entidades físicas, a partir de tres criterios: 1) que las entidades físicas se pueden tocar y ver, en oposición a las entidades mentales; 2) que tienen existencia pública; y, 3) que su existencia es constante, no desaparece. Para esto, se les mostraron dos imágenes cuyo contenido contrasta; en la primera imagen, está un personaje que posee una galleta y, en la segunda, un personaje que piensa en una galleta. Se le pregunta al niño cuál de estas entidades puede ser tocada y vista por otra persona o manipulada en el futuro.

Los niños de tres, cuatro y cinco años son muy acertados en estos juicios, le asignan un estatus ontológico acertado a las entidades físicas: son públicas y se pueden ver, diferenciándolas de las entidades mentales; es decir, que no dan muestra de tendencias al realismo ontológico (Wellman, 1995). Wellman y Estes (1986), creen que es posible que el niño no comprenda "pensar" como una experiencia mental, sino que el niño interprete que "el personaje que piensa en la galleta" simplemente "es un personaje que no tiene una galleta". Estos resultados confirman que, al contrario del "realismo infantil" propuesto por Piaget, los niños de tres años comprenden la distinción entre entidades mentales y reales y, lo que es más importante, no son realistas en el plano más sutil de confundir las entidades mentales con las físicas intangibles como el humo, las sombras o los sonidos; sin embargo, no lo hacen con la misma consistencia que los niños de cuatro y cinco años.

Después de abordar la comprensión por parte de los niños de las entidades mentales de un personaje, Estes et al. (1989) deciden examinar, en primer lugar, las concepciones que el niño tiene de sus propios estados mentales y, en segundo lugar, debido a que los resultados de las anteriores investigaciones son inconsistentes con el realismo ontológico, se interroga al niño sobre imágenes mentales a la manera de "fotografías en nuestra cabeza", que son elementos de representación que lo invitan a concebir las imágenes mentales como fotografías físicas reales y pueden llevarlo a concepciones realistas erróneas.

Los resultados que exhiben los niños de tres años, sin ser iguales a los de cuatro años, son con- cluyentes. Responden positivamente a las preguntas que indagan por la transformación de las fotografías mentales, y negativamente a las transformaciones de los objetos reales. Las respuestas a los ítems ver, tocar y publicidad sobre las imágenes mentales se dieron dentro de cuatro categorías mutuamente excluyentes: 1) mental: se alude al término mental en la explicación "Solo lo estoy pensando en mi mente"; 2) no real: la entidad no puede ser vista o tocada por otra persona porque no es real; 3) inaccesible: la entidad es inaccesible "Esta dentro de mi cabeza"; y, 4) imposible: no se puede realizar la acción "Nadie puede hacer eso".

Wellman (1995) argumenta que es probable que el rendimiento de los niños enmascare una sutil comprensión de los términos mentales, que les permita contestar acertadamente aunque carezcan de un concepto adecuado de procesos mentales como pensar. Por otro lado, Piaget (1929 citado en Wellman, 1995) explica que el uso de términos mentales como "pensar" por parte de los niños no significa que los niños sean concientes de la distinción entre ideas y cosas. Entonces, en este experimento se elimina el uso de términos mentales en las instrucciones como "piensa", por, "intenta dibujar un balón en tu cabeza", esperando ofrecer evidencias frente a la afirmación de que el rendimiento de los niños en las tareas se debe a una comprensión superficial e incorrecta de los términos mentales.

En las investigaciones que indagan por la comprensión de los niños de la deseabilidad, se encuentra que el niño entiende la relación entre el deseo y los resultados, y reconocen que el logro de una meta conduce a emociones placenteras como la felicidad, y su frustración, a la tristeza. Indagaciones más recientes (Peerbhoy, 1990 citado en Perner, 1994) demuestran que los niños de tres años consideran que personaje con malas intenciones estará triste, mientras que el niño de cinco años juzga que estará feliz. Los niños de tres años razonan que el personaje estará triste porque consideran la deseabilidad una característica objetiva (la deseabilidad de la meta hace que se vea como un hecho objetivo). Sin embargo, Flavell et al. (1990 citados en Perner, 1994), demuestran que los niños comprenden el deseo como una relación muy per- 
sonal con las cosas. Los niños construyen modelos complejos necesarios para representar el deseo de una situación particular y el modo en que éste se relaciona con las metas logradas (Perner, 1994).

\section{Relación entre el desarrollo de Teoría de la Mente y el desarrollo del lenguaje}

En relación con el desarrollo del lenguaje y el cognitivo, el punto de vista clásico comentado en la literatura es el de Piaget, señala que el desarrollo conceptual precede al semántico. El niño desarrolla, en primer lugar, los conceptos y luego les asigna una etiqueta (Gopnik \& Meltzoff, 1999). En las últimas décadas, han surgido diversas teorías que explican la adquisición infantil del lenguaje, entre ellas se encuentran diversas posturas que van desde la afirmación de que el lenguaje precede el desarrollo cognitivo, a una postura opuesta que considera que el desarrollo del lenguaje depende del desarrollo cognitivo.

Gopnik y Meltzoff (1999) señalan la existencia de una relación bidireccional entre los desarrollo del lenguaje y el desarrollo cognitivo, y sugieren que estas habilidades pueden ser importantes para la comprensión misma de la falsa creencia, lo cual apoya el argumento de que los niños desarrollan una Teoría de la Mente integrada. Lo cierto es que, durante este periodo que comprende hasta aproximadamente los 5 años de edad, es el tiempo en el cual se desarrolla una teoría de la mente y es el periodo de mayor adquisición del lenguaje, el cual, sin duda, permite establecer algunas relaciones. Desde esta perspectiva, la adquisición del lenguaje es entendida como un proceso análogo al desarrollo científico, aportando un modelo útil para el estudio de la relación entre las habilidades lingüísticas y conceptuales.

Desde el punto de vista de Gopnik y Meltzoff (1999), una de las perspectivas sólidas dentro de la teoría-teoría, se establece que el desarrollo semántico y cognitivo emergen simultáneamente; ninguno de los dos precede al otro, por el contrario avanza paralelamente, cada área facilita a la otra desde los inicios del lenguaje. Pero, esta interacción depende en sí misma de los logros cognitivos pre- vios y también de los problemas de orden cognitivo que afronten los niños en ese momento.

El desarrollo cognitivo no solo es un prerrequisito para el desarrollo semántico, sino que también lo motiva. Cuando los niños solucionan un problema conceptual particular, la atención del niño se dirige hacia las palabras relevantes para estos problemas, pero el descubrimiento de la naturaleza de los patrones lingüísticos le permite a su vez resolver dichos problemas y dirigir la atención hacia otros nuevos. A su vez, la conducta no lingüística (clasificación) es paralela a la conducta lingüística de ubicar los objetos en diferentes categorías. Sin embargo, los niños aprovechan las señales lingüísticas solamente si las consideran relevantes pera los problemas específicos que desean resolver (Gopnik \& Meltzoff, 1999). Dentro de la estructura central teórica, el niño utiliza las palabras que necesita para codificar los conceptos relacionados.

A los 18 meses de edad, se presenta en el niño lo que varios autores han llamado de manera gráfica la "explosión de la denominación” (Bates, 1979; Goldfield \& Reznick, 1990). Para Goldfield y Reznick, este aumento de vocabulario consiste enteramente en nombrar objetos, al mismo tiempo que el niño exhibe actuaciones exhaustivas de seriación y clasificación de los mismos. Pero las relaciones especificas entre las palabras de denominación y los tres dominios cognitivos primordiales, como la permanencia de los objetos, los medios-fines y las categorías de los objetos, comparten características comunes: 1) las tres tienen lugar al mismo tiempo, alrededor de los 18 meses; y, 2) las tres implican desarrollos semánticos particulares, pero son completamente independientes entre sí.

En general, los niños adquieren la expresión "no está" y las habilidades no lingüísticas relacionadas con la permanencia de los objetos, meses antes de adquirir las expresiones de éxito/fracaso y las relacionadas con habilidades medios/fines, pero otros niños no siguen estos patrones, por el contrario los invierten. Estos patrones temporales aportan las pruebas más sólidas para la relación bidireccional entre lenguaje y cognición, ya que demuestran la estrecha y específica relación entre el lenguaje y la cognición no lingüística. Sin embargo, los resulta- 
dos de los estudios translingüísticos sugieren que es la adquisición de información, específicamente la información lingüística, el factor crucial para el desarrollo conceptual; el niño que escucha el lenguaje relevante para un problema particular que está afrontando, tendrá más probabilidad de resolver el problema que los niños que no disponen de las palabras necesarias para codificar los conceptos relacionados, aspecto que apoya las teorías de la modularidad.

Otro aspecto importante es que los datos reportados en un estudio longitudinal realizado por Astington y Jenkins (1999) apoyan el argumento de Villiers (2000) de que es la sintaxis, una función estructural del lenguaje de particular importancia, la que provee el formato representacional necesario para la comprensión de la falsa creencia. Sin embargo, no existe convencimiento total de que sea la adquisición de las estructuras sintácticas específicas las que producen dicha comprensión, dado que Custer (1996 citado en Astington \& Jenkins, 1999) concluye que se requiere algo más que las estructuras, para la adquisición de la Teoría de la Mente. Sin embargo, Astington y Jenkins si dejan claro que aunque los resultados de su investigación no son concluyentes, sí son consistentes con la aseveración de que es el desarrollo del lenguaje el que precede el desarrollo cognitivo.

Por último, Gopnik y Meltzoff (1999) recalcan que las pruebas aportadas se encuentran entre las pocas demostraciones empíricas que muestran que en la infancia existe una estrecha interacción entre el desarrollo semántico y el desarrollo conceptual; que emergen simultáneamente en un lapso específico, y que son captadas ampliamente por la perspectiva de la teoría-teoría, ya que a la hora de aprender una teoría, el cambio semántico y conceptual van de la mano. No obstante, enfatizan que no todo el lenguaje tiene una relación tan íntima con el desarrollo conceptual; existen palabras que están más relacionadas con rutinas sociales y guiones; además, como ya se expresó, los estudios translingüísticos sugieren que los aspectos de la entrada de información lingüística puede tener efectos sobre el desarrollo conceptual; igualmente, los estudios transculturales y de diferencias individuales pueden arrojar algo de luz sobre esta relación. Pero también es importante resaltar que para estas autoras, "la información lingüística es simplemente una fuente más de resultados, pero en este aspecto el niño nuevamente no se diferencia en nada de un científico moderno que se fía de los informes de otros para casi toda su base empírica" (p. 210).

\section{El presente estudio}

Con el propósito de precisar la naturaleza de dicha relación entre el desarrollo semántico del lenguaje y el desarrollo de la comprensión de las falsas creencias, se establecen tres hipótesis entre los desempeños en las tareas de la falsa creencia y la habilidad del lenguaje: 1) que A depende de B; 2) que B depende de A; y, 3) que ambas, A y B dependen de un tercer factor $\mathrm{C}$.

La primera hipótesis se apoya en los hallazgos según los cuales el desarrollo del lenguaje precede el desarrollo de la Teoría de la Mente. El desarrollo del lenguaje provee a los niños de recursos tanto de habilidad sintáctica como de comprensión semántica, que promueven y permiten una comprensión de la falsa creencia. No existe evidencia que indique que los niños comprenden la representación de las creencias antes de que dispongan de los medios lingüísticos para expresar su comprensión (Astington \& Jenkins, 1999).

En la segunda hipótesis, se propone que el desarrollo de la teoría es un facilitador del lenguaje siendo consistentes con la visión de Piaget, Wellman o Perner (1994), según los cuales, solo la actividad representacional permite la comprensión de las tareas de la falsa creencia; de hecho, el adulto que pierde la capacidad del lenguaje no pierde la capacidad de comprender las creencias falsas. Esta hipótesis también es consistente con la perspectiva propuesta por Leslie que argumenta que la comprensión de la falsa creencia es dependiente de sistemas modulares innatos.

La tercera hipótesis supone que los medios del lenguaje y la teoría de la mente se correlacionan porque los dos dependen de otro factor interno como la memoria o la función ejecutiva, que le 
permite al niño razonar usando reglas fijas más complejas que son entonces usadas en las tareas del lenguaje y en la teoría de la mente, o por factores externos como las actividades culturales y sociales.

En un estudio longitudinal, los niños de 3 años se pusieron a prueba tres veces (al comienzo, a la mitad y al final) en un periodo de tiempo de 7 meses. Se aplicó un test de desarrollo del lenguaje; paralelamente se aplicaron tres tareas sobre creencias que requieren de las habilidades lingüísticas: la tarea clásica de la falsa creencia y la tarea de contenidos inesperados de Wimmer y Perner (1983) y la tarea de apariencia y realidad de Flavell et al. (1983). Astington y Jenkins (1999) encuentran que las habilidades tempranas del lenguaje predicen el desempeño de los niños en las tareas de la falsa creencia, y que los desempeños bajos en los test del lenguaje no lo predicen, coincidiendo con el argumento que sostiene que el lenguaje es fundamental para el desarrollo de la teoría de la mente.

A lo largo de la investigación, la competencia del lenguaje se incrementó firmemente a través del tiempo en los tres momentos en que se evaluó, y el desempeño de la Teoría de la Mente se incrementó en los tiempos uno y dos, y después se niveló. Las correlaciones de las calificaciones en las tareas de creencia y el test de lenguaje, aunque no son significativas en cada medición, siempre estuvieron en la dirección esperada por Astington y Jenkins (1999).

Frente a los anteriores resultados, las investigadoras profundizan en la manera en que los aspectos pragmáticos, semánticos y sintácticos del lenguaje, están relacionados con el desarrollo de la teoría de la mente y encuentran que aunque los puntajes de semántica y sintaxis están altamente correlacionados, la sintaxis hace una contribución independiente a la predicción sobre la aparición de la Teoría de la Mente. Aunque no son concluyentes los resultados, los datos sugieren que es la sintaxis, una función estructural del lenguaje de particular importancia, la que provee el formato representacional necesario para la comprensión de la falsa creencia, como lo expresan de Villiers y de Villiers (2000), quienes argumentan que el dominio de las estructuras de complemento sintáctico es lo que provee el formato representacional necesario para la comprensión de la falsa creencia.

Sin embargo, en otra investigación, Custer (1996 citado en Astington \& Jenkins, 1999) expone que los niños no tienen el mismo desempeño en tareas que incluyen oraciones con igual complemento de objeto, por ejemplo: "El niño esta fingiendo que su cachorrito está afuera" y "El niño piensa que su cachorrito está afuera". Los niños aprueban la tarea cuando se usa fingir y fallan cuando se emplea pensar, lo que permite concluir que se requiere algo más que las estructuras sintácticas para la adquisición de la Teoría de la Mente.

Por último, Astington y Jenkins (2001) dejan claro que el lenguaje es central para el desarrollo de la Teoría de la Mente, debido a que le provee de un andamiaje efectivo para las representaciones simbólicas (metarrepresentaciones), que son lo suficiente bien elaboradas como para invalidar otras interpretaciones generadas por la experiencia directa; sin embargo, no comparten el total convencimiento de que sea la adquisición de las estructuras sintácticas específicas la que procure esta comprensión en los niños.

\section{Método}

\section{Diseño de la investigación}

Para esta investigación, se propone realizar un estudio de tipo transversal descriptivo, puesto que no es posible de antemano asignar aleatoriamente una trayectoria que demuestre la estrecha y específica relación entre el desarrollo de las habilidades lingüísticas y las habilidades cognitivas en los niños. No obstante, se busca establecer los patrones correlaciónales o causales entre las distintas variables (Hernández, Fernández \& Baptista, 1998).

También es posible que existan terceros factores que puedan ser responsables de las relaciones aparentes entre el desarrollo lingüístico y cognitivo, que no son susceptibles de control como lo es la inteligencia general y el género. El estudio empírico de los cambios semánticos asociados al cambio cognitivo, son de dominio específico e independientes unos de otros, a diferencia de los cambios 
estructurales globales, lo que permite abordar los efectos de las variables ocultas de forma diferente. Se pueden comparar los logros de un niño en un dominio y contrastarlos con los logros de ese mismo niño en otros dominios.

\section{Participantes}

Fueron 116 niños de jardines infantiles, provenientes todos de clase media alta, de las áreas metropolitana de Bogotá y Chía -según los criterios de estratificación del Departamento Administrativo Nacional de Estadística (DANE)-, distribuidos en tres grupos así: 36 niños (16 niños y 20 niñas) en un rango de edad de 1 año y 10 meses a 2 años 4 meses, con una media de 2 años, pertenecientes al grado Prejardín; 38 niños (18 niños y 20 niñas) en un rango de edad de 2 años y 10 meses a 3 años 4 meses, con una media de 3 años, pertenecientes al grado Jardín A; y, 42 niños (24 niños y 18 niñas) en un rango de edad de 3 años y 10 meses a 4 años 4 meses, con una media de 4 años, pertenecientes al grado Transición. El proceso de selección de los niños se realizó a través de un muestreo no probabilístico por conveniencia de un grupo de niños y se estableció, como criterio de exclusión, la presencia de dificultades en el desarrollo lingüístico o cognitivo.

\section{Materiales}

\section{Tareas de Falsa Creencia}

El desarrollo de investigaciones empíricas con niños de 2 a 4 años, implica el uso de instrumentos que les permita actuar y responder de forma natural. Las situaciones experimentales presentadas consisten en una plataforma digital, desarrollada en el programa Flash, de las tres versiones clásicas de las tareas de la falsa creencia, diseñadas, originalmente, con el objetivo de evaluar la capacidad de los sujetos para atribuir creencias falsas a los demás (véase anexo para cada situación).

Para la tarea clásica de la falsa creencia de Wimmer y Perner (1983), se narra la historia de un personaje que llega del colegio y guarda una galleta en un frasco. Su madre lo cambia de lugar. Se interroga al niño en dónde buscará el personaje la galleta. Para la tarea del recipiente engañoso de Perner et al. (1987) se emplearon tres cajas familiares con contenidos inesperados: 1) una caja de fósforos que contiene un lápiz dentro; 2) una caja de crayones que contiene un reloj; y, 3) una caja de leche que contiene una muñeca. Para la tarea de apariencia y realidad de Flavell (1983), se emplearon dos objetos que parecen ser una cosa pero que realmente son otra: 1) una esponja que parece una piedra, y 2) un lápiz que parece una culebra de juguete.

\section{Lenguaje}

Se empleó la batería de evaluación de desarrollo temprano del lenguaje (Hresko et al., 1981) para evaluar las habilidades semánticas del lenguaje. La batería mide la habilidad general del lenguaje por medio de destrezas semánticas y sintácticas, a través de las respuestas a preguntas específicas; consta de seis subescalas, de las cuales se utilizaron dos: la de comprensión lexical y la de competencia semántica. El manual de la batería designa individualmente cada punto como perteneciente a categorías sintácticas y semánticas. El número de puntos representa cada constructo, permitiendo determinar empíricamente la dificultad y el poder discriminativo de los puntos. La batería ha sido estimada en niños entre 2 y 7 años de edad, con buena integridad en el test-retest, buena consistencia interna y buena estabilidad.

\section{Tarea de no deseo}

Para la Tarea de no deseo, se narra una historia sin de creencias ni deseos, en donde se muestra al personaje llegando del colegio y dejando el maletín en la casa.

\section{Procedimiento}

El estudio fue dividido en dos fases para evaluar el desempeño entre el desarrollo lingüístico y el desarrollo de la Teoría de la Mente: 1) se evaluó 
el desarrollo de las habilidades lingüísticas en el aspecto semántico, mediante la aplicación de la batería de desarrollo temprano del lenguaje; y, 2) se aplicaron tres tareas de falsa creencia con el propósito de evaluar la capacidad de los niños para atribuir falsas creencias.

El desarrollo de las distintas tareas se llevó a cabo en una habitación amplia bien iluminada, con una mesa y dos sillas apropiadas al tamaño de los niños. La situación se registró con cámara de video. Cada niño se evaluó individualmente mediante el batería del desarrollo temprano del lenguaje, en las tres tareas sobre las creencias falsas y en la tarea de no deseo. En una sección particular, el experimentador presentó las instrucciones de cada una de las tareas. A cada una de las tareas, se le instauraron controles de procedimiento como contrabalanceo en la presentación de las tareas y en el orden de las preguntas de control. Las preguntas de control permiten verificar si el niño falla en sus respuestas debido a que ha olvidando algunos aspectos trascendentales de la historia.

En primera instancia, el niño es introducido a una historia protagonizada por un par de muñecos que involucra diferentes situaciones, con el propósito de familiarizarlo con los distintos ambientes experimentales y con el tipo de interacción que se le plantea.

1. El niño asiste a un escenario compuesto por dos muñecas y dos recipientes en el que se representa la siguiente escena: un muñeco guarda una tableta de chocolate dentro de uno de los dos recipientes y luego sale de la habitación a jugar. Durante su ausencia la madre cambia de forma imprevista la ubicación del chocolate del recipiente $\mathrm{A}$, hacia el recipiente $\mathrm{B}$. Entonces, el muñeco regresa por su chocolate. En seguida, el experimentador realiza la pregunta de la tarea sobre la falsa creencia "iDonde piensa Juan que está el chocolate?", y las preguntas de control "¿Dónde colocó Juan el chocolate al principio?".
2. En la tarea del recipiente engañoso con contenidos inesperados de Perner et al. (1987), a las niños se les pregunta por el contenido familiar de la caja. Los niños responden haciendo alusión a los contenidos usuales, como se espera. En seguida, se abre la caja y se muestra al niño el contenido inusual que hay dentro. Entonces, el experimentador indaga al niño por lo que él creía que había en la caja antes de ser abierta “¿Qué creías que había dentro?”, y por lo que otro niño que no ha visto el contenido de la caja cree que contiene "¿Qué creería Juanito que hay dentro?". Igualmente, se realizan las preguntas de control "iPuedes recordar que hay aquí adentro?".

3. En la tarea de apariencia y realidad de Flavell (1983), a los niños se les muestra un objeto que parece una cosa, pero que realmente es otra (una esponja que parece una piedra). Se deja al niño manipular el objeto para descubrir su identidad, lo que realmente es. A los niños se les pregunta primero por la apariencia del objeto "iEl objeto "parece" una piedra o una esponja?" y, en seguida, por lo que realmente es el objeto " $i E l$ objeto es realmente una piedra o una esponja?”.

Si cualquiera de las preguntas en los test de falsa creencia no se responde espontáneamente, al niño se le da una alternativa de elección forzada.

\section{Resultados}

En el presente estudio se exploraron las asociaciones entre el desempeño de una muestra de 116 niños entre los 2 a 4 años en cuanto a la comprensión social, por medio de tres versiones de la tarea de la falsa creencia, junto con dos subpruebas de la escala de evaluación del lenguaje temprano, específicamente, la de desarrollo léxico y la de competencia semántica. En la Tabla 1 se encuentran los descriptivos para cada tarea y escalas. 
TABLA 1

Medias y desviaciones estándar de cada una de las mediciones

\begin{tabular}{lcc}
\hline \multicolumn{1}{c}{ Tarea /Escala } & Media & DE \\
\hline Tarea de Cambio Inesperado & 0,36 & 0,48 \\
Tarea de Recipiente Engañoso & 0,31 & 0,46 \\
Tarea de Apariencia Realidad & 0,17 & 0,37 \\
Escala de Desarrollo Léxico & 10,49 & 2,63 \\
Escala de Competencia Semántica & 10,91 & 3,82 \\
\hline
\end{tabular}

Fuente: elaboración propia

En conjunto, los niños obtuvieron un mejor desempeño en la tarea de cambio inesperado que en las otras dos versiones de la tarea de falsa creencia. Por otra parte, su desempeño en la escala de competencia semántica fue mejor que en la escala de desarrollo léxico.

En la Figura 1 encontramos los porcentajes por rango de edad en cada una de las tareas de falsa creencia.

Tal y como refleja la gráfica anterior, se percibe un patrón creciente con la edad en cada una de las tareas; sin embargo, los desempeños fueron mucho más bajos para la tarea de apariencia realidad que en las otras dos versiones. A fin de analizar diferencias de promedios entre edades para cada tarea, se realizó un ANOVA unifactorial. Los datos son recogidos en la Tabla 2 .

\section{TABLA 2}

ANOVA para las tres tareas de Falsa Creencia de acuerdo al rango de edad

\begin{tabular}{cccc}
\hline Tipo de Tarea & $g l$ & $F$ & $p$ \\
\hline Cambio Inesperado & $(2,115)$ & 7,93 & 0,00 \\
Recipiente Engaños & $(2,115)$ & 9,05 & 0,00 \\
Apariencia Realidad & $(2,115)$ & 3,78 & 0,02 \\
\hline
\end{tabular}

$P<0,05$

Fuente: elaboración propia

Debido a que los valores $F$ fueron significativos con un nivel de significancia $<0,05$. Se procedió a realizar la prueba post hoc de Tukey para evaluar diferencias honestamente significativas entre cada rango de edad, en las tres tareas. Los datos están recogidos en las Tablas 3, 4 y 5.

\section{FIGURA 1}

Resultados de los niños por rango de edad para cada una de las tareas

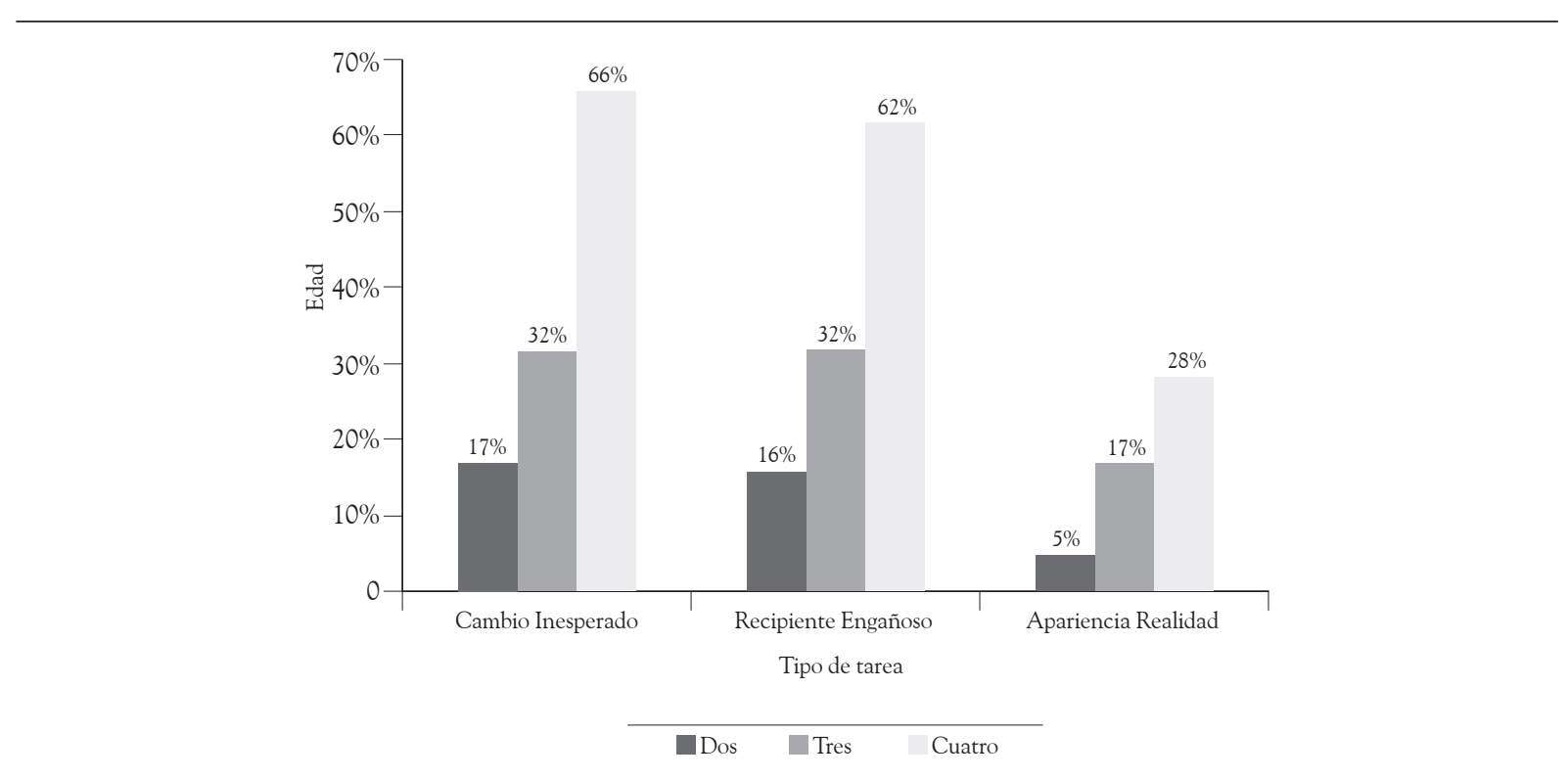

Fuente: elaboración propia 
TABLA 3

Análisis post hoc de Tukey para la Tarea Cambio Inesperado por Rango de Edad

\begin{tabular}{ccccc}
\hline \multirow{2}{*}{$\begin{array}{c}\text { Rango } \\
\text { de Edad }\end{array}$} & \multicolumn{2}{c}{3} & \multicolumn{2}{c}{4} \\
\cline { 2 - 5 } & $\begin{array}{c}\text { Diferencia } \\
\text { de Medias }\end{array}$ & $\begin{array}{c}\text { Signifi- } \\
\text { cación }\end{array}$ & $\begin{array}{c}\text { Diferencia } \\
\text { de Medias }\end{array}$ & $\begin{array}{c}\text { Signifi- } \\
\text { cación }\end{array}$ \\
\hline 2 & 0,15 & 0,34 & 0,40 & $0,00 * *$ \\
3 & & & 0,26 & $0,04 * *$ \\
\hline
\end{tabular}

$P<0,05$

Fuente: elaboración propia

Para esta tarea se encontraron diferencias significativas entre las edades de 2 y 4 , y 3 y 4 años. No se encontraron diferencias significativas entre las edades de 2 y 3 años.

TABLA 4

Análisis post hoc de Tukey para la Tarea Recipiente Engañoso por Rango de Edad

\begin{tabular}{ccccc}
\hline \multirow{2}{*}{$\begin{array}{c}\text { Rango } \\
\text { de Edad }\end{array}$} & \multicolumn{2}{c}{3} & \multicolumn{2}{c}{4} \\
\cline { 2 - 5 } & $\begin{array}{c}\text { Diferencia } \\
\text { de Medias }\end{array}$ & $\begin{array}{c}\text { Signifi- } \\
\text { cación }\end{array}$ & $\begin{array}{c}\text { Diferencia } \\
\text { de Medias }\end{array}$ & $\begin{array}{c}\text { Signifi- } \\
\text { cación }\end{array}$ \\
\hline 2 & 0,15 & 0,29 & 0,41 & $0,00 * *$ \\
3 & & & 0,26 & $0,02 * *$ \\
\hline
\end{tabular}

$P<0,05$.

Fuente: elaboración propia

Para esta tarea se encontraron diferencias significativas entre las edades de 2 y 4 , y 3 y 4 años. No se encontraron diferencias significativas entre las edades de 2 y 3 años.

\section{TABLA 5}

Análisis post hoc de Tukey para la Tarea Apariencia Realidad por Rango de Edad

\begin{tabular}{ccccc}
\hline \multirow{2}{*}{$\begin{array}{c}\text { Rango } \\
\text { de Edad }\end{array}$} & \multicolumn{2}{c}{3} & \multicolumn{2}{c}{4} \\
\cline { 2 - 5 } & $\begin{array}{c}\text { Diferencia } \\
\text { de Medias }\end{array}$ & $\begin{array}{c}\text { Signifi- } \\
\text { cación }\end{array}$ & $\begin{array}{c}\text { Diferencia } \\
\text { de Medias }\end{array}$ & $\begin{array}{c}\text { Signifi- } \\
\text { cación }\end{array}$ \\
\hline 2 & 0,10 & 0,46 & 0,23 & $0,02 * *$ \\
3 & & & 0,13 & 0,27 \\
\hline
\end{tabular}

Fuente: elaboración propia
Para esta tarea no se encontraron diferencias significativas entre las edades de 2 y 4 , y 3 y 4 años. Se encontraron diferencias significativas entre las edades de 2 y 4 años.

Con respecto al género, se evaluaron las cuatro tareas utilizando la prueba $t$ para grupos relacionados, encontrándose, en la tarea de cambio inesperado un valor de $t=8,08(\mathrm{p}=0,36)$; en la tarea de recipiente engañoso un valor de $t=7,19$ $(p=0,31)$ y para la tarea de apariencia realidad un valor de $t=4,90(p=0,17)$. En síntesis, no se encontraron diferencias de género para las tareas de falsa creencia.

\section{Análisis de Asociaciones entre Tareas de Falsa Creencia y Desarrollo Semántico del Lenguaje}

En la Figura 2 se presentan los valores promedio obtenidos por los niños en cada una de las edades para las escalas de lenguaje semántico evaluados.

\section{FIGURA 2}

Desempeño promedio en las escalas de comprensión lexical y competencia semántica

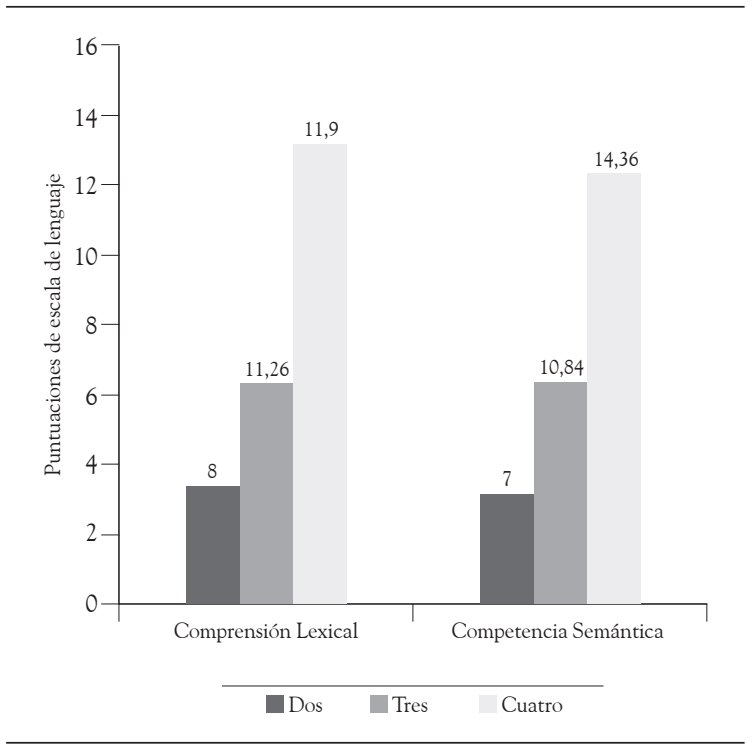

Fuente: elaboración propia

A excepción del rango de edad de 2 años, la competencia semántica obtuvo un desempeño promedio superior a la de comprensión lexical. 
En la Tabla 6 se presenta la matriz de correlaciones para las tres tareas de falsa creencia y para las dos escalas de desarrollo semántico.

\section{TABLA 6}

Matriz de Correlaciones de las variables Falsa Creencia y Desarrollo Semántico

\begin{tabular}{|c|c|c|c|c|c|}
\hline & Variable & 2 & 3 & 4 & 5 \\
\hline \multirow{3}{*}{$\begin{array}{l}\text { Falsa } \\
\text { Creen- } \\
\text { cia }\end{array}$} & $\begin{array}{l}\text { 1. Cambio } \\
\text { Inesperado }\end{array}$ & $0,89^{* * *}$ & $0,61^{* *}$ & 0,17 & $0,24^{* *}$ \\
\hline & $\begin{array}{l}\text { 2. Recipiente } \\
\text { Engañoso }\end{array}$ & 1 & $0,68^{* *}$ & 0,17 & $0,25^{* *}$ \\
\hline & $\begin{array}{l}\text { 3. Apariencia } \\
\text { Realidad }\end{array}$ & & 1 & 0,09 & 0,08 \\
\hline $\begin{array}{l}\text { Desa- } \\
\text { rrollo }\end{array}$ & $\begin{array}{l}\text { Comprensión } \\
\text { Lexical }\end{array}$ & & & 1 & $0,67^{*}$ \\
\hline $\begin{array}{l}\text { Semán- } \\
\text { tico }\end{array}$ & $\begin{array}{l}\text { 5. Competen- } \\
\text { cia Semántica }\end{array}$ & & & & 1 \\
\hline
\end{tabular}

$* \pi<0.05$

$* * \pi<0.01$

Fuente: elaboración propia

Se encuentran asociaciones significativas entre las tareas de cambio inesperado y recipiente engañoso, entre las tareas de cambio inesperado y apariencia realidad. De igual manera, se encuentran asociaciones significativas entre las tareas de cambio inesperado y recipiente engañoso, con la escala de competencia semántica, y entre las dos escalas que evalúan desarrollo semántico del lenguaje. A fin de explorar las posibles influencias entre las variables comprensión emocional (evaluado en las tres versiones de la tarea de falsa creencia) y desarrollo semántico del lenguaje (evaluado en las dos subescalas de lenguaje), se procedió a realizar un análisis de regresión que tuviera como independiente el desarrollo la comprensión de la falsa creencia y como dependiente el desarrollo semántico del lenguaje, encontrándose un modelo de $R^{2}=0,065$ con valor de $F\left({ }_{4}\right)=2,61 p=0,055$. No dio significativo este modelo.

Posteriormente, se ensayó un modelo en donde el desarrollo semántico del lenguaje se tomaba como independiente y como dependiente, el desarrollo de la comprensión de la falsa creencia, encontrándose en modelo de $R^{2}=0,046$ con valor de $F\left({ }_{4}\right)=5,46 p=0,021$ significativo al 0,05. Es decir, la varianza de la comprensión de la falsa creencia es explicada en un $21 \%$ por la varianza del desarrollo semántico del lenguaje.

\section{Discusión}

En la presente investigación, se han encontrado evidencias empíricas que apoyan la hipótesis de que el desarrollo de la comprensión de las teorías infantiles de la mente, es subsidiaría del desarrollo semántico del lenguaje en niños de 3 a 4 años edad. En éste sentido, los hallazgos del presente estudio son consistentes con los encontrados, entre otros, con las investigaciones realizadas por Astington (2001) y Astington \& Baird (2005) en el sentido de la primacía del desarrollo lingüístico sobre el desarrollo sociocognitivo.

Los hallazgos señalan una dirección, contrario a lo propuesto por Gopnik y Meltzoff (1999), que señalan la existencia de una relación bidireccional entre los desarrollo del lenguaje y el desarrollo cognitivo, y sugieren que estas habilidades pueden ser importantes para la comprensión misma de la falsa creencia, lo cual apoya el argumento de que los niños desarrollan una Teoría de la Mente integrada. En ese orden de ideas, los hallazgos resultan coherentes con otras evidencias en otras áreas del lenguaje como el desarrollo de la sintaxis (de Villiers \& de Villiers, 2000).

Por otra parte, el uso de la plataforma digital, en el presente estudio, se constituye en una alternativa válida en la evaluación de las Teorías Infantiles de la Mente, que aportan y enriquecen la discusión sobre el uso de las tareas de Falsa Creencia como elemento que permite señalar o no la presencia de Teoría de la Mente.

En las investigaciones de las últimas décadas, la tarea clásica de la falsa creencia se ha tomado como indicador de la posesión de una Teoría de la Mente a los 4 años, momento para el cual, se manifiesta un cambio radical en la comprensión de la mente. Bloom (2000) aporta dos razones fundamentales para abandonar la tarea de la falsa creencia como 
prueba para el desarrollo de la Teoría de la Mente: 1) la tarea de la falsa creencia requiere de otras habilidades para razonar sobre ella; y, 2) la Teoría de la Mente no necesariamente exige la habilidad para razonar sobre ella.

Bloom (2000) señala que para solucionar la tarea de la falsa creencia, el niño tiene que seguir la acción de dos vías de narración, observar el intercambio del chocolate, recordar donde se colocó y apreciar el significado preciso de la pregunta, y aunque las preguntas de control aseguren que el niño de 3 años puede hacer frente a las demandas básicas de la tarea, los niños fallan. En las tareas simplificadas, las modificaciones permiten desempeños más favorables, bajando la edad de quienes pasan la tarea.

En investigaciones posteriores, Wellman, Cross y Watson (2001) realizan un metaanálisis de las respuestas o juicios correctos sobre las falsas creencias por parte de los niños en muchas de las tareas (591 condiciones de falsas creencias) que indagan el desarrollo de la Teoría de la Mente, permitiendo organizar los hallazgos disponibles acerca del entendimiento de falsas creencias.

Wellman et al. (2001) evaluaron un conjunto de factores que varían a través de los distintos estudios, encontrando que los resultados en las tareas de la falsa creencia se agrupan sistemáticamente, con algunas pocas excepciones; más aún, desde el punto de vista metodológico, el desempeño de los niños en las tareas de falsa creencia informa acerca de un gran número de variaciones que son esencialmente equivalentes, mostrando un patrón consistente: los juicios de los niños progresan de desempeños desfavorables a desempeños más exitosos, resultados que son consistentes con los enfoques teóricos que proponen que el entendimiento de la creencia exhibe un genuino cambio conceptual alrededor de los 4 años.

Los resultados del presente estudio no sólo señalan que las tareas de falsa creencia siguen siendo un indicador confiable de la presencia de Teoría de la Mente en niños, sino que además el uso de los medios digitales favorece la comprensión y apropiación de las tareas de comprensión de las teorías de la mente, constituyéndose en otra puerta de entrada al universo sociocognitivo del niño.

\section{Referencias}

Astington, J. W.; Harris, P. \& Olson, D. (Eds). (1988). Developing theories of mind New York: Cambridge University Press.

Astington, J. W. (1997). "Theory of mind" goes to school. Educational Leadership, 56 (3), 46-48.

Astington, J. W. (1998). El descubrimiento infantil de la mente. Madrid: Morata.

Astington, J. W. (2001). The future of Theory-of-Mind: Understanding motivational states, the role of language, and real-world consequences. Child Development, 72, 685-687.

Astington, J. W. \& Jenkins, J. M. (1999). A longitudinal study of the relation between language and Theory-of-Mind development. Development Psychology, 35, 1311-1320.

Astington, J. \& Baird, J. (2005). Why language matters for Theory of Mind. London: Oxford University Press.

Baron-Cohen, S., Leslie, A. \& Frith, U. (1985). Does the autistic child have a theory of mind. Cognition, 21, 37-46.

Baron-Cohen, S., Tager-Flusberg, H. \& Cohen, D. (Eds). (1993) Understanding others minds: perspectives from autism. Oxford: Oxford University Press.

Bartsch, K. and Wellman, H. M. (1989). Young children's attribution of action to beliefs and desires. Child Development, 60, 946-964.

Bloom, P. \& German, T. (2000). Two reason for abandon the false belief task as a theory of mind. Cognition, 77, 25-31.

Bruner, J. (1986). El habla del niño. Barcelona: Paidós.

Chandler, M. (1988). Doubt and developing theories of mind. En J. Astington, P. Harris \& D. Olson (Eds.), Developing theories of mind, pp. 387-413. New York: Cambridge University Press.

Chandler, M., Firtz, A. and Hala, S. (1989). Small-scale deceit: Deception as a marker of two, three and four year olds early theories of mind. Child Development, 60, 1263-1277.

de Villiers, J. \& de Villiers, P. A. (2000). Linguistic determinism and the understanding of false beliefs. In P. Mitchell \& K. Riggs (Eds.), Children's reasoning and the mind (pp. 191-228). Hove, UK: Psychology Press. 
Engel, P. (1993). Psicología ordinaria y ciencia cognitiva. Barcelona: Gedisa.

Fodor, J. A. (1983). La modularidad de la mente. Madrid: Morata

Gómez, J. C. (1991). Visual behavior as a window for reading the mind of others in primtates, en A. Whiten (comp) Natural theories of mind: Evolution, development and simulation of everyday mindreading, pp. 195-207. Cambridge: Basil Blackwell.

Gopnik, A (1993). How we know our minds: The illusion of first-person knowledge of intentionality. Behavioral and Brain Sciences, 16, 1-15, 90-101

Gopnik, A. \& Meltzoff, A. (1999). Palabras, pensamientos y teorías. Madrid: Visor.

Gopnik, A. y Meltzoff, A. (2002) What children will teach scientists. In J. Brockman (Ed.) The next fifty years: Science in the first half of the twenty-first century, pp. 62-73. New York: Vintage

Harris, P.L. (1989). Children and Emotion: the development of psychological understanding. Oxford: Basil Blackwell.

Harris, P.L. (1991). The work of the imagination. en A. Whiten (comp) Natural theories of mind: Evolution, development and simulation of everyday mindreading. Cambridge: Basil Blackwell.

Harris, P.L. (1992). From simulation to folk psychology: the case for development. Mind and Language, 7(1-2), 120-144.

Harris, P.L. (1993). Pretending and planning. En S. Baron- Cohen, H. Tager-Flusberg \& D. Cohen (eds). Understanding Other Minds (pp. 228-245). Oxford: Oxford University.

Hirschfeld, L. y Gelman, S. (2002). Cartografía de la mente. Barcelona: Paidós Vol. II

Humphrey, N. (1986). La mirada interior. Madrid: Alianza editores.

Humphrey, N. (1987). La reconquista de la conciencia. México: Fondo de cultura económico.

Karmiloff-Smith, A. \& Inhelder, B. (1974). If you want to get ahead, get a theory. Cognition, 3, 195-212.

Leslie, A. (1987). Pretense and representation: The origins of "Theory of Mind". Psychological Review, 94, 412-426.

Leslie, A. (1991). Pretense, autism and the theory of mind module. Current Directions in Psychological Science, 6, 315-324.
Martí, E. (1997). Construir una mente. Madrid: Paidós.

Pérez - Pereira, M. (1995). Nuevas perspectivas en la psicología del desarrollo. Madrid: Alianza.

Perner, J. (1994). Comprender la mente Representacional. Madrid: Alianza.

Piaget, J. (1929). La representación del mundo en el niño. Madrid: Morata.

Premack, D. \& Woodruff, G. (1978). Does the chimpancé have a theory of mind?. Behavioral and Brain Sciences, 1, 515-526.

Premack, D. y Premack, A. (1983). La mente del simio. Madrid: Debate Madrid.

Puche, R. (2000). Formación de herramientas cientificas en el niño pequeño. Bogota: Arango Editores, Universidad del Valle.

Rivière, A. \& Núñez, M. (1996). La mirada mental. Buenos Aires: AIQUE.

Sattler, J. (1996). Evaluación infantil. Mexico: Manual Moderno.

Wellman, H, \& Woolley, J.D. (1986). From simples desires to ordinary beliefs: the early development of everyday psychology. Cognition, 35, 245-275

Wellman, H. y Bartsch K. (1988). Young child's reasoning about beliefs. Cognition, 30, 239-277.

Wellman, H. (1995). Desarrollo de la teoría del pensamiento en los niños. Bilbao: Desclée de Brouwer.

Wellman, H. \& Estes, D. (1986). Early understanding of mental: A reexamination of childhood realism. Child Development, 57, 910-932.

Wellman, H., Cross, D. \& Watson, J. (2001). Metaanalysis of Theory-of-Mind development: The truth about false belief. Child Development, 72, 655-684.

Whiten, A. (comp) (1991). Natural theories of mind: Evolution, development and simulation of everyday mindreading. Cambridge: Basil Blackwell.

Wimmer, H. \& Perner, J. (1983). Beliefs about beliefs: Representation and constraining function of wrong beliefs in young children's understanding of deception. Cognition, 13, 103-128.

Wimmer, H. \& Weichbold, V. (1994). Children's theory of mind: Fodor's heuristics examined. Cognition, 53, 45-57.

Zaitchik, D. (1991). ¿Is only seeing really believing?: Sources of the true belief in the false belief task. Cognition Development, 6, 91-103. 
